\title{
On the displacement in origin for turbulent boundary layers subjected to sudden changes in wall temperature and roughness
}

\author{
Mila R. Avelino ${ }^{\text {a }}$, Atila P. Silva Freire ${ }^{\mathrm{b}, *}$ \\ a Department of Mechanical Engineering (UERJ), Rua São Francisco Xavier, 524, sala 5023, Bloco A, 20550-013 Rio de Janeiro, Brazil \\ b Mechanical Engineering Program (PEM/COPPE/UFRJ), Federal University of Rio de Janeiro, CP 68503, \\ 21945-970 Rio de Janeiro, Brazil
}

Received 18 February 2001; received in revised form 8 January 2002

\begin{abstract}
The present work studies the behavior of flows that develop over surfaces that present a sudden change in surface temperature and roughness. A particular interest of this study is to investigate any existing relationship between the error in origin for both the velocity and the temperature profiles, so that any analogy between the logarithmic laws for the velocity and the temperature profiles can be assessed. Three different types of surfaces are considered and the flow is made to pass from a cold smooth surface to a hot rough surface. Measurements are presented for the mean velocity and temperature profiles. (C) 2002 Elsevier Science Ltd. All rights reserved.
\end{abstract}

Keywords: Turbulence; Thermal boundary layer; Roughness; Error in origin

\section{Introduction}

A classical means to enhance the transfer of heat at a wall is to use surfaces that are not smooth but rough. Then, depending on the geometry of the roughness elements, the transfer of heat can be altered at will. In fact, the problem of selecting surfaces that will furnish a determined heat transfer coefficient to a particular application is not a trivial one.

In previous studies of flows over rough surfaces, different methods have been used to construct the roughness. The early studies have used sand grains glued onto a surface. The more recent studies have preferred machine protrusion with a well-defined geometry. In the latter case, authors (see, e.g., [1-4]) have classified the rough surfaces into two distinct types of surfaces: (1) $K$ type rough surfaces, and (2) $D$ type rough surfaces. In cases where the nature of the roughness can be expressed

\footnotetext{
${ }^{*}$ Corresponding author. Tel.: +55-21-2562-7748; fax: $+55-$ 21-2562-7748.

E-mail address: atila@serv.com.ufrj.br (A.P. Silva Freire).
}

with the help of a single length scale, the height of the protrusions, $K$, the surface is termed of type $K$. Flows, on the other hand, which are apparently insensitive to the characteristic scale $K$, but depend on other global scale of the flow are termed $D$ type flows. This is the case when the roughness is geometrically characterized by a surface with a series of closely spaced grooves within which the flow generates stable vortical configurations.

Naturally, most of the studies on flows over rough surfaces have dealt so far with the velocity field. Indeed, the complexities caused by the roughness on the proper assessment of the flow properties are of such an order that, even today, after the advent of very sophisticated measuring techniques, much still remains to be understood about the problem. That is the reason why not many works on the temperature field are available in the literature. The result is that, for the evaluation of properties related to the thermal boundary layer, the standard approach is to resort to some analogy between the momentum and the heat transfer processes. For simple flow situations, such as flows in the completely developed regime, these approaches are seen to provide good results. For example, the classical result $C_{\mathrm{f}} / 2=S t$, 


\begin{tabular}{|c|c|c|c|}
\hline \multicolumn{4}{|c|}{ Nomenclature } \\
\hline$A$ & parameter in velocity law of the wall & \multicolumn{2}{|c|}{ Greek symbols } \\
\hline$B$ & parameter in temperature law of the wall & $\delta$ & boundary layer thickness \\
\hline$C_{i}$ & parameter in velocity law of the wall & $\delta^{*}$ & boundary layer displacement thickness \\
\hline$D_{i}$ & parameter in temperature law of the wall & $\varepsilon$ & error in origin \\
\hline$C_{\mathrm{f}}$ & skin-friction coefficient $=1 / 2\left(u_{\tau}^{2} / U_{\infty}^{2}\right)$ & $\theta$ & boundary layer momentum thickness \\
\hline$G$ & Clauser parameter defined by Eq. (9) & $\Theta$ & non-dimensional temperature $=\left(T_{\mathrm{w}}-t\right) /$ \\
\hline K & height of roughness element & & $\left(T_{\mathrm{w}}-T_{\infty}\right)$ \\
\hline $\operatorname{Pr}$ & Prandtl number & $x$ & von Karman's constant $(=0.4)$ \\
\hline$P$ & pressure & $\lambda$ & distance between leading edge of roughness \\
\hline $\operatorname{Re}$ & Reynolds number & & elements \\
\hline$S$ & length of roughness elements & $\mu$ & viscosity \\
\hline St & Stanton number $=u_{\tau} t_{\tau} / U_{\infty}\left(T_{\mathrm{w}}-T_{\infty}\right)$ & $v$ & kinematic viscosity \\
\hline$T, t$ & temperature & $\Pi$ & Coles's wake profile \\
\hline$\Delta t$ & roughness function defined by Eq. (6) & $\rho$ & density \\
\hline$t_{\tau}$ & friction temperature & $\tau$ & shear stress \\
\hline$U, u$ & longitudinal velocity component & \multirow{2}{*}{\multicolumn{2}{|c|}{ Subscripts }} \\
\hline$u_{\tau}$ & friction velocity & & external flow conditions \\
\hline$\Delta u$ & roughness function defined by Eq. (2) & e & $\begin{array}{l}\text { external flow conditions } \\
\text { temperature }\end{array}$ \\
\hline$x, y$ & flow cartesian coordinates & w & $\begin{array}{l}\text { temperature } \\
\text { conditions at the wall }\end{array}$ \\
\hline$y_{\mathrm{T}}$ & $\begin{array}{l}\text { distance measured from the crest of rough- } \\
\text { ness elements }\end{array}$ & $\infty$ & external flow conditions \\
\hline$W$ & $\begin{array}{l}\text { gap between roughness elements, also } \\
\text { Coles's function }\end{array}$ & & \\
\hline
\end{tabular}

where $C_{\mathrm{f}}$ is the skin-friction coefficient and $S t$ is the Stanton number, is a very good working expression which has been used extensively in the past.

The purpose of this work is to investigate both the velocity and the temperature fields of boundary layer flows that develop over surfaces with a sudden change in roughness. In the cases of interest to be studied here, a cold flow over a smooth surface is made to pass over a hot, rough surface. Thus, for a certain length after the change in surface nature, the velocity and the thermal boundary layers will be in a different state of development. In this situation, it is not clear that a straight Reynolds analogy will work. Here we are especially interested in studying the validity of two universal relations, the law of the wall and the law of the wake, for both, the velocity and the temperature fields.

For flows over a rough surface, we know that $C_{\mathrm{f}}$ and $S t$ cannot be evaluated directly through methods that resort to the gradient of the log-law because the effective origin of the boundary layer is not known a priori. This prompted some authors (e.g., $[1,5])$ to develop detailed procedures for the determination of this effective origin that could be used to evaluate $C_{\mathrm{f}}$ directly from the angular coefficient of a "corrected" law of the wall.

In this work, the behavior of the error in origin for the velocity and the temperature fields will be investigated for three types of rough surfaces. Then, any analogy between the velocity and the temperature fields will be assessed. To achieve that, the present work will investigate experimentally the characteristics of turbulent boundary layers that are subjected to step changes in surface roughness and temperature, with emphasis on the characterization of the inner layer velocity and temperature profiles.

Over the years, several studies on the behavior of boundary layers having a non-uniform distribution of temperature or heat flux at the wall were carried out. For flows over smooth walls, the works of Hartnett et al. [6], Johnson [7,8], Reynolds et al. [9,10] and Spalding [11] are classical. Johnson [7] reports that for a thermal boundary layer with $4.27 \mathrm{~m}$ of unheated starting length and a free-stream velocity of $7.62 \mathrm{~m} / \mathrm{s}$, measurements taken $1.83 \mathrm{~m}$ downstream of the step point reveal that the normalized temperature profiles have shapes different from the normalized velocity profile. Also, the temperature intermittence profile has a different form than the velocity intermittence profile. Antonia et al. [12] considered $1.83 \mathrm{~m}$ of unheated length, after which a constant surface heat flux was applied. He observed that after $1.8 \mathrm{~m}$ of development the temperature profiles had not yet reached a fully developed form.

For flows over rough surfaces, the number of works is much smaller. The Heat and Mass Transfer Group at Stanford University has been very active in the last three 
decades, having published a number of reference works. Studies on flows over rough surfaces with changes in the thermal boundary conditions were made by Coleman et al. [13] and by Ligrani et al. [14-16]. With the help of a kernel function and the superposition of a heat transfer theory, expressions were advanced for the evaluation of Stanton number which were supposed to hold for such different conditions as variable wall temperature, wall blowing and free-stream velocity, and steps in wall temperature and blowing.

Here, we intend to provide further experimental data that can be used to characterize flows over surfaces with a non-uniform distribution of roughness and temperature at a wall. The data, in particular, will be used to test the theory presented in [17-19].

\section{Experimental procedure}

The experiments were carried out in the high-turbulence wind tunnel located in the Laboratory of Turbulence Mechanics of PEM/COPPE/UFRJ. The tunnel is an open circuit tunnel with a test section of dimensions $67 \mathrm{~cm} \times 67 \mathrm{~cm} \times 3 \mathrm{~m}$ (Fig. 1). The test section is divided into three sections of equal length which can be fitted with surfaces having different types of roughness and of wall heating. The first section, which is normally kept at ambient temperature, consists of a smooth glass wall. The second and third parts of the test section are equipped with independent electric heaters.

The flow was subjected to a step change in roughness after travelling the first meter over the glass wall. Three types of rough surfaces were considered where the roughness elements consisted of equally spaced transversal rectangular slats. The dimensions of the roughness elements are shown in Table 1 where $K$ denotes the height, $S$ the length, $W$ the gap, and $\lambda$ is the pitch. In constructing the surface, extreme care was taken to keep the first roughness element always depressed below the smooth surface, its crest kept aligned with the smooth glass wall surface.

The glass surface was always kept at $25 \pm 0.5^{\circ} \mathrm{C}$. The next two meters, fitted with the rough surfaces, had their temperature raised to $75 \pm 3{ }^{\circ} \mathrm{C}$. In fact, the variation in surface temperature for most of the plates was very

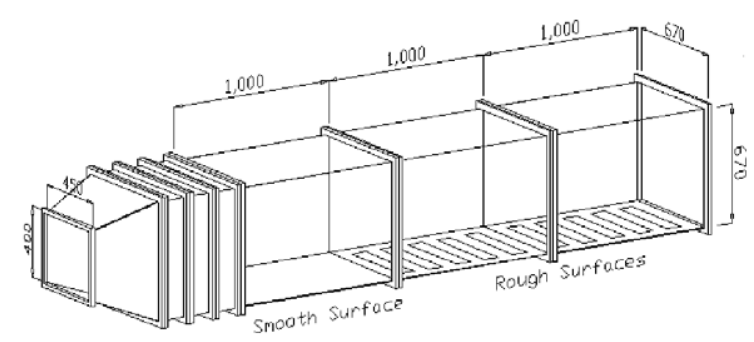

Fig. 1. Wind tunnel geometry. Dimensions in millimeter.
Table 1

Geometry of the roughness elements

\begin{tabular}{llllll}
\hline Type & $K(\mathrm{~mm})$ & $S(\mathrm{~mm})$ & $W(\mathrm{~mm})$ & $\lambda(\mathrm{mm})$ & $W / K$ \\
\hline I & 4.77 & 15.88 & 15.88 & 31.76 & 3.33 \\
II & 4.77 & 15.88 & 31.76 & 47.64 & 6.66 \\
III & 6.35 & 4.76 & 15.88 & 20.64 & 2.5 \\
\hline
\end{tabular}

small, within $\pm 1{ }^{\circ} \mathrm{C}$. However, the plates were manufactured in such a way that, at the junction (over a length of $10 \mathrm{~cm}$ ), conjugated effects resulted in a small decrease in temperature $\left(-3{ }^{\circ} \mathrm{C}\right)$. The wall temperature was controlled by 15 thermocouples, set at five streamwise stations at three span-wise positions. Because the wind tunnel was an open circuit tunnel, controlling the temperature in the final $0.1 \mathrm{~m}$ was difficult (in the very last measuring section a decrease of $5{ }^{\circ} \mathrm{C}$ in mean temperature was observed).

The measurements were performed for values of the free-stream velocity of $3.12 \mathrm{~m} / \mathrm{s}$; the free-stream level of turbulence was about $2 \%$. The stream-wise pressure gradient was closely set to zero by adjusting the roof of the tunnel according to the readings of eight equally spaced pressure taps.

Mean velocity profiles and turbulence intensity levels were obtained using a DANTEC hot-wire system series $56 \mathrm{~N}$. The boundary layer probe was of the type 55P15. A Pitot tube, an electronic manometer, and a computer controlled traverse gear were also used. In getting the data, 10000 samples were considered. The profiles were constructed from about 100 points. The mean temperature profiles were obtained through a chromelconstantan micro-thermocouple mounted on the same traverse gear system used for the hot-wire probe. An uncertainty analysis of the data was performed according to the procedure described in [20]. The uncertainty associated with the velocity and temperature measurements was: $U=0.064 \mathrm{~m} / \mathrm{s}$ precision, 0 bias $(P=0.95)$; $T=0.214{ }^{\circ} \mathrm{C}$ precision, 0 bias $(P=0.99)$.

To obtain accurate measurements, the mean and fluctuating components of the output signal of the anemometer were treated separately. Two output channels of the anemometer were used. The mean velocity profiles were calculated directly from the untreated signal of channel one. The signal given by channel two was $1 \mathrm{~Hz}$ high-pass filtered leaving, therefore, only the fluctuating velocity. The later signal was then amplified with a gain controlled between 1 and 500 and shifted by an offset so as to adjust the amplitude of the signal to the range of the $\mathrm{A} / \mathrm{N}$ converter.

\section{Theory}

Before considering the experimental data, let us first introduce a short review on the theory of turbulent flow over rough surfaces. 
For this type of flow, Moore [21] has shown that a universal expression can be written for the wall region provided the origin for measuring the velocity profile is set some distance below the crest of the roughness elements. The displacement in origin is normally referred to in the literature as the error in origin, $\varepsilon$. A detailed method to determine the displaced origin can be found originally in [1] and more recently in [5].

Thus, for any kind of rough surface, it is possible to write

$\frac{u}{u_{\tau}}=\frac{1}{x} \ln \left[\frac{\left(y_{\mathrm{T}}+\varepsilon\right) u_{\tau}}{v}\right]+A-\frac{\Delta u}{u_{\tau}}$,

where

$\frac{\Delta u}{u_{\tau}}=\frac{1}{x} \ln \left[\frac{\varepsilon u_{\tau}}{v}\right]+C_{i}$

and $x=0.4, A=5.0$, and $C_{i}, i=K, D$, is a parameter characteristic of the roughness (see, e.g., [1]).

Eqs. (1) and (2), although of a universal character, have the inconvenience of needing two unknown parameters for their definition, the skin-friction velocity, $u_{\tau}$, and the error in origin, $\varepsilon$. A chief concern of many works on the subject is, hence, to characterize these two parameters.

In fact, the fundamental concepts and ideas on the problem of a fluid flowing over a rough surface were first established by Nikuradse [22] who investigated the flow in sand-roughened pipes. Even at that early age, Nikuradse was capable to establish that, at high Reynolds number, the near wall flow becomes independent of viscosity, being a function of the roughness scale, the pipe diameter and Reynolds number. He also found that, for the defect layer, the universal laws apply to the bulk of the flow irrespective of the conditions at the wall. The roughness effects are, therefore, restricted to a thin wall layer.

Thus, considering that Coles's wake hypothesis [23] applies to the outer region of the flow, the law of the wall can be re-written as

$\frac{u}{u_{\tau}}=\frac{1}{x} \ln \left[\frac{\left(y_{\mathrm{T}}+\varepsilon\right) u_{\tau}}{v}\right]+A-\frac{\Delta u}{u_{\tau}}+\frac{\Pi}{x} W\left(\frac{y}{\delta}\right)$,

where $W$ is a universal function of $y / \delta$ and $\Pi$ is a parameter dependent on the upstream shear stress and pressure distribution.

Eq. (3) provides a representation of the velocity field over the whole of the turbulent and defect regions of the boundary layer.

Substitution of $(y, u)=\left(\delta, U_{\infty}\right)$ into Eq. (3) furnishes

$\frac{U_{\infty}}{u_{\tau}}=\frac{1}{\chi} \ln \left[\frac{\delta+\varepsilon}{\varepsilon}\right]+A-C_{i}+\frac{2 \Pi}{\chi}$.

This is a simple algebraic equation that furnishes values of $C_{\mathrm{f}}\left(=2 u_{\tau}^{2} / U_{\infty}^{2}\right)$ for known values of $U_{\infty}, \delta$ and $\varepsilon$.
Having established Eq. (4) for the velocity boundary layer, we now want to advance an analogy for the temperature boundary layer.

To extend Eqs. (1) and (2) to the temperature turbulent boundary layer we will use the theory of Silva Freire and Hirata [17]. Alternatively, we could have used dimensional arguments. The details of the theory will be omitted; here it suffices to say that, from an asymptotic point of view, the important factor in the determination of the flow structure is the correct assessment of the order of magnitude of the fluctuating quantities. Then, analogies between the transfer of momentum and the transfer of heat can be constructed.

For flows over rough surfaces, we have seen that the characteristic length scale for the near wall region must be the displacement in origin. Indeed, in this situation, the viscosity becomes irrelevant for the determination of the inner wall scale because the stress is transmitted by pressure forces in the wakes formed by the crests of the roughness elements. It is also clear that, if the roughness elements penetrate well into the fully turbulent region, then the displaced origin for both the velocity and the temperature profiles will always be located in the overlap fully turbulent region. The similarity in transfer processes for turbulent flows then suggests that

$\frac{T_{\mathrm{w}}-t}{t_{\tau}}=\frac{1}{x_{\mathrm{t}}} \ln \left[\operatorname{Pr} \frac{\left(y_{\mathrm{T}}+\varepsilon_{\mathrm{t}}\right) u_{\tau}}{v}\right]+B-\frac{\Delta t}{t_{\tau}}$,

where

$\frac{\Delta t}{t_{\tau}}=\frac{1}{x_{\mathrm{t}}} \ln \left[\operatorname{Pr} \frac{\varepsilon_{\mathrm{t}} u_{\tau}}{v}\right]+D_{i}$

and $D_{i}, i=K, D$, is a constant characteristic of the roughness.

Eqs. (5) and (6) are the law of the wall formulation for flows over rough surfaces with transfer of heat.

To describe the temperature profile in the defect region of the boundary layer, we may consider that Coles's wake hypothesis also holds for the temperature field so that Eq. (3) may be re-written as

$\frac{T_{\mathrm{w}}-t}{t_{\tau}}=\frac{1}{x_{\mathrm{t}}} \ln \left[\operatorname{Pr} \frac{\left(y_{\mathrm{T}}+\varepsilon_{\mathrm{t}}\right) u_{\tau}}{v}\right]+B-\frac{\Delta t}{t_{\tau}}+\frac{\Pi_{\mathrm{t}}}{x_{\mathrm{t}}} W\left(\frac{y}{\delta_{\mathrm{t}}}\right)$,

where the wake profile $\Pi_{\mathrm{t}}$ should, in principle, be a function of the enthalpy thickness.

This equation provides a representation for the temperature field that can be allowed to sustain a different state of development from the velocity field. As a result, Stanton number can be evaluated independently from the skin-friction through a particular equation. To find this equation, we substitute $(y, t)=\left(\delta_{\mathrm{t}}, T_{\infty}\right)$ into Eq. (7) to get 
$\frac{T_{\infty}-T_{\mathrm{w}}}{t_{\tau}}=\frac{1}{\chi_{\mathrm{t}}} \ln \left[\operatorname{Pr} \frac{\left(\delta_{\mathrm{t}}+\varepsilon_{\mathrm{t}}\right)}{\varepsilon_{\mathrm{t}}}\right]+B-D_{i}+\frac{2 \Pi_{\mathrm{t}}}{\chi_{\mathrm{t}}}$.

This algebraic equation can now be used to find Stanton number as a function of $T_{\infty}, \delta_{\mathrm{t}}$ and $\varepsilon$.

A comment seems now in order. If a direct Reynolds analogy was to hold between the velocity and temperature fields, then for the non-dimensional values of $u_{\tau}$ and of $t_{\tau}$ furnished by Eqs. (5) and (7) to be identical, it would be necessary that all constants and parameters appearing in the equations should have close values. Thus, for all types of rough surfaces, the error in origin for both the velocity and the temperature profiles should have close values. That would ultimately mean that the temperature displacement in origin could be determined from the dynamic characteristics of the flow.

\section{Experiments}

\subsection{Velocity profile data}

The measured velocity profiles for the three different flow configurations are shown in Fig. 2. It is clear from this figure that the logarithmic regions of the flow have suffered a slight deformation to the left side. In fact, as we shall see, a very popular method to find $\varepsilon$ is based on

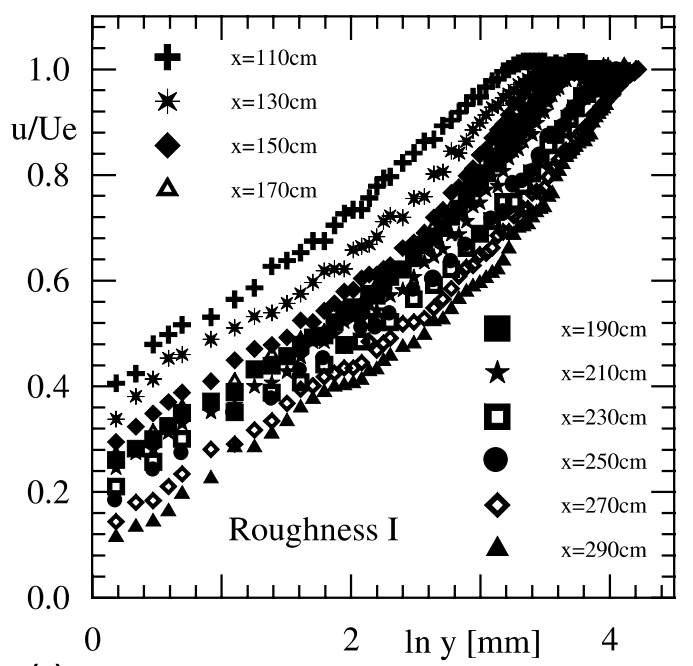

(a)

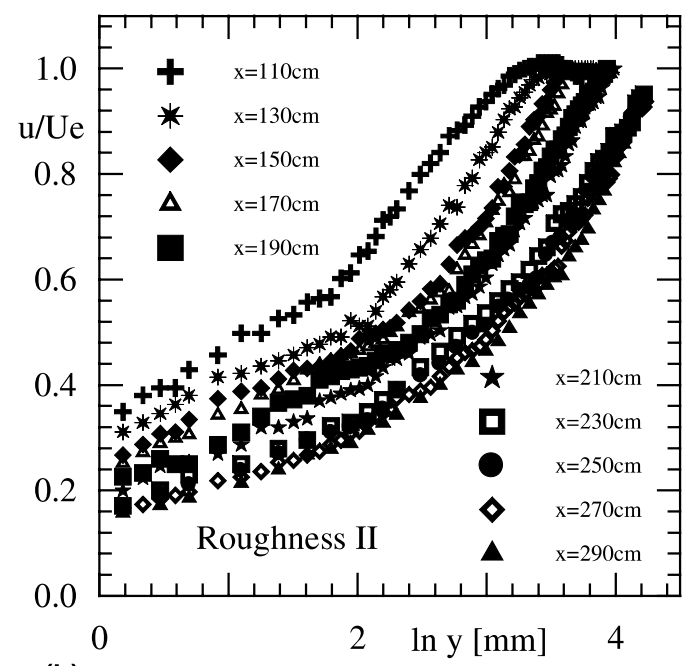

(b)

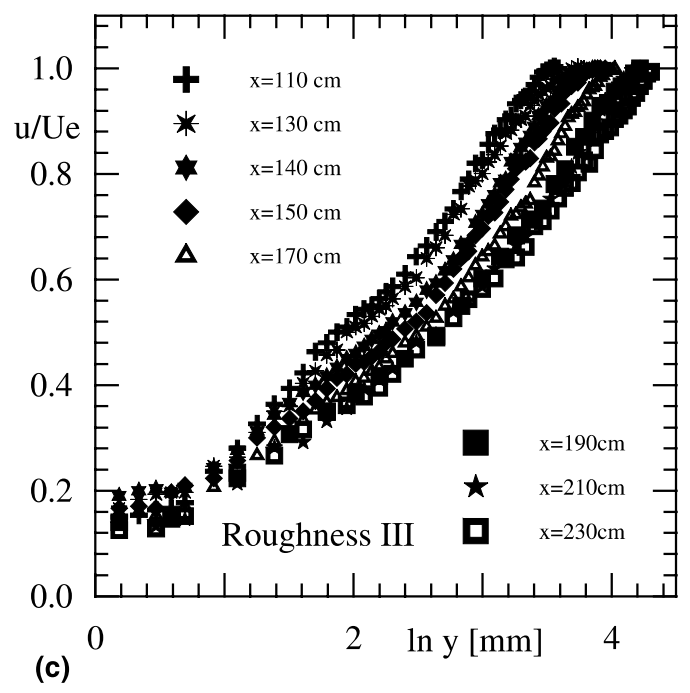

Fig. 2. Velocity profiles: (a) roughness of type I; (b) roughness of type II; (c) roughness of type III. 


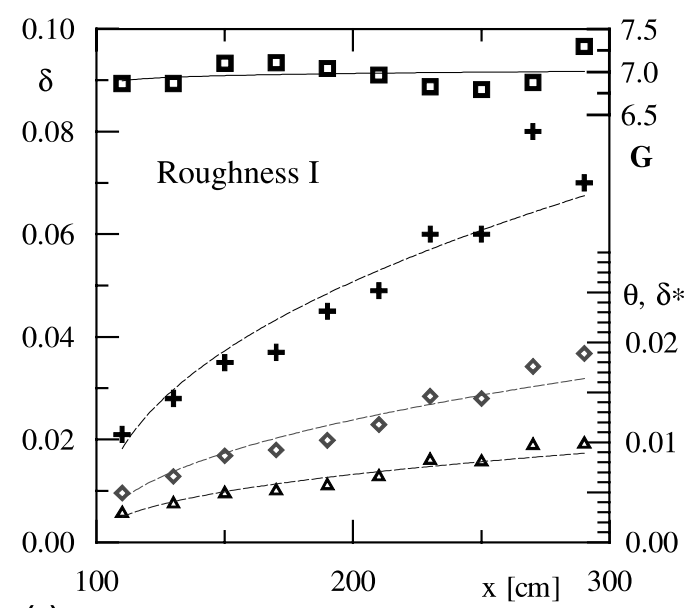

(a)

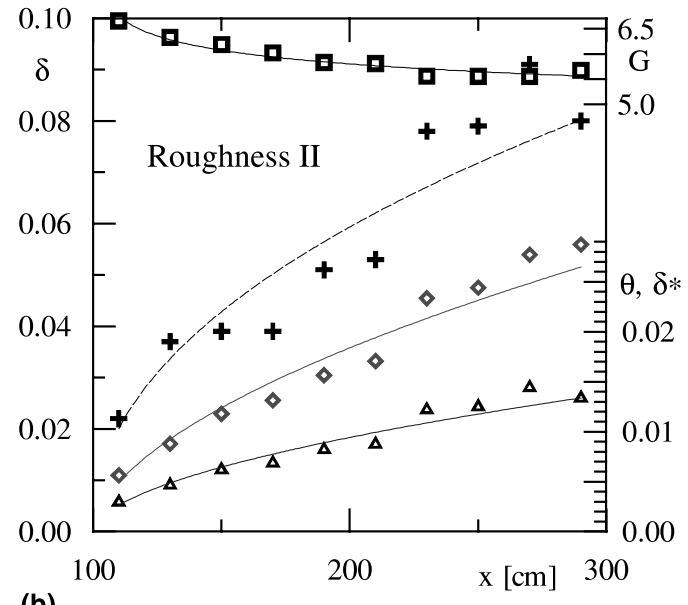

(b)

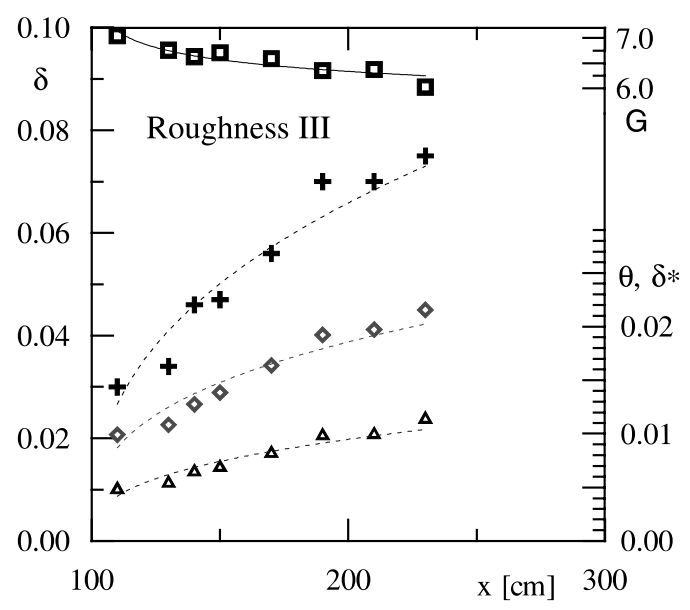

(c)

Fig. 3. Global parameters: (a) roughness of type I; (b) roughness of type II; (c) roughness of type III. (+) boundary layer thickness; $(\diamond)$ displacement thickness; $(\Delta)$ momentum thickness; $(\square)$ Clauser factor.

a procedure to restore the lower portion of the velocity profile to a logarithmic profile.

The global parameters of the velocity boundary layers are shown in Fig. 3, where $\delta$ denotes the boundary layer thickness, $\delta^{*}$ the displacement thickness and $\theta$ is the momentum thickness. Of particular note are the results for the Clauser factor, $G$, defined by

$G=\frac{1}{\Delta} \int_{0}^{\infty}\left(\frac{U_{\mathrm{e}}-\bar{u}}{u_{\tau}}\right)^{2} \mathrm{~d} y$,

where

$\Delta=\int_{0}^{\infty} \frac{U_{\mathrm{e}}-\bar{u}}{u_{\tau}} \mathrm{d} y=\delta^{*} \sqrt{2 / C_{\mathrm{f}}}$.

This parameter indicates the state of equilibrium of the boundary layer. For the values found here, between
6.0 and 7.0, the boundary layer is in a self-preserving state. Please note that the evaluation of $G$ depends on the knowledge of $C_{\mathrm{f}} / 2$ which, in principle, is not known at the moment. The determination of $C_{\mathrm{f}} / 2$ is explained in the following.

The error in origin, $\varepsilon$, was estimated by four different procedures. In fact, the procedures of Perry and Joubert [1] and of Perry et al. [5] are the most rigorous that can be found in the literature so that the data resulting from them must be seen as very reliable. The procedures of Thompson [24] and of Bandyopadhyay [25] are very simplified so that the values of $\varepsilon$ obtained through them must be seen just as a first approximation.

In the Perry and Joubert [1] method, arbitrary values of $\varepsilon$ are added to the wall distance measured from the top of the roughness elements and a straight line is fitted 
to the log-law region. The value of $\varepsilon$ that furnishes the best discriminated logarithmic region is then considered to be the correct value for the error in origin. The method of Perry et al. [5] is more sophisticated, resorting to a cross plot of $\varepsilon$ vs $2 \Pi / x$, where $\Pi$ stands for Coles's wake profile.

Thus, to determine the error in origin, the velocity profiles were plotted in semi-log graphs in dimensional coordinates. Next, the normal distance from the wall was incremented by $0.1 \mathrm{~mm}$ and a straight line fit was applied to the resulting points. The best fit was chosen by searching for the maximum coefficient of determination, R-squared. Other statistical parameters were also observed, the residual sum of squares and the re- sidual mean square. Normally, a coefficient of determination superior to 0.99 was obtained.

Having found $\varepsilon$, we can now use the gradient of the log-law to determine $u_{\tau}$. Other method to obtain $u_{\tau}$ is the momentum-integral equation. This latter method, however, is very sensitive to any three-dimensionality of the flow and the determination of the derivatives of the various mean flow parameters is a highly inaccurate process.

The difficulty with both cited methods is that they depend on the evaluation of derivatives. For flows subjected to step changes in surface roughness, the momentum-integral method further suffers from the ill definition of the boundary layer origin. The process of
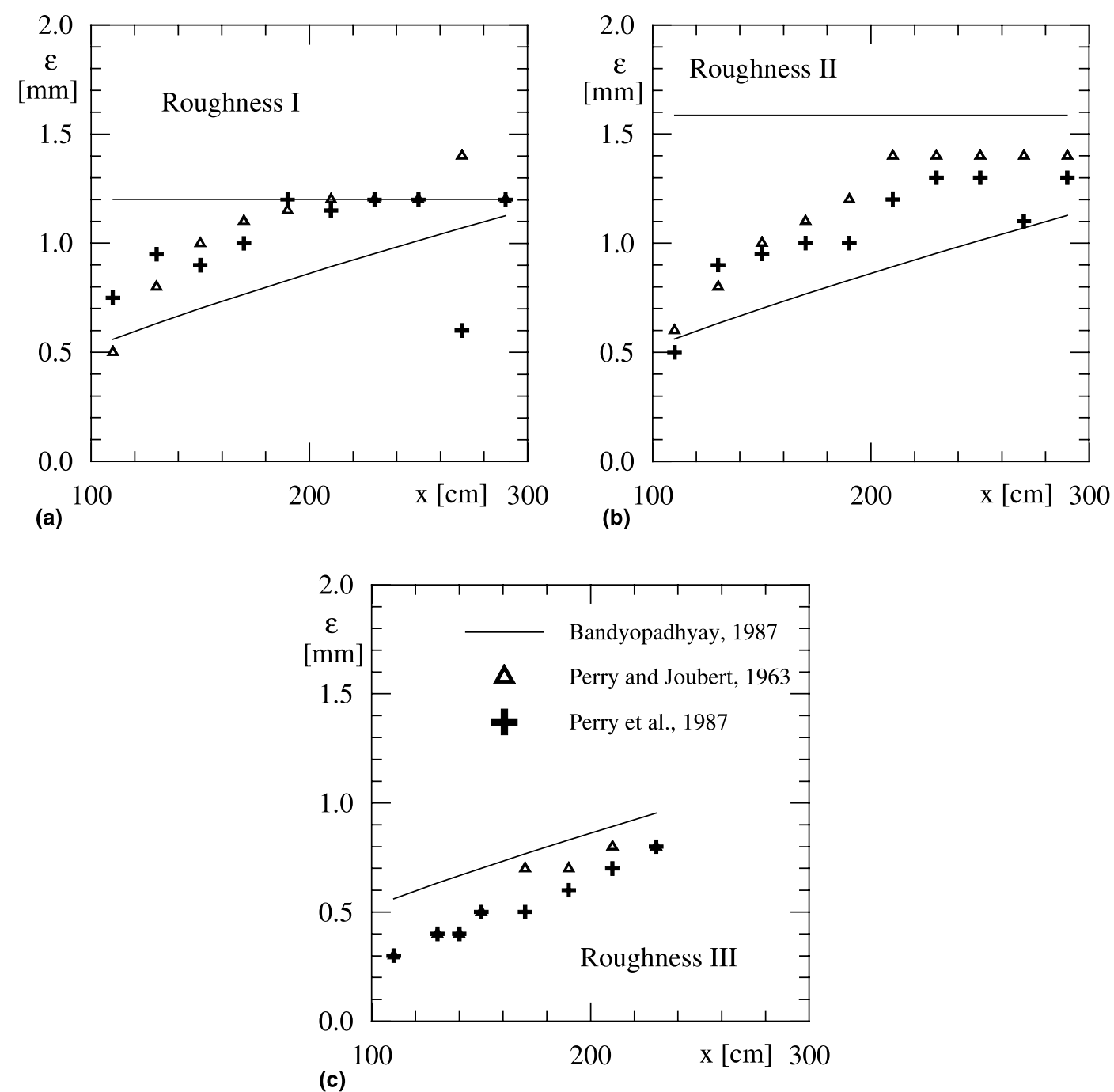

Fig. 4. Error in origin for the velocity profiles: (a) roughness of type I; (b) roughness of type II; (c) roughness of type III. $(\Delta)$ [1], $(+)$ [5]; (thin line) Thompson [24]; (thick line) [25]. 
finding adequate parameters for a good curve fitting is, therefore, highly aggravated.

The $\varepsilon$ results for the rough surfaces of types, I, II and III, are presented in Fig. 4. Considering the high degree of difficulty involved in finding these results, and the very good agreement between the predictions based on the two more reliable procedures, we may say that the results of $\varepsilon$ and consequently of $C_{\mathrm{f}}$ are likely to be very representative of the flow.

Fig. 4 clearly shows that $\varepsilon$ presents a relatively quick stream-wise evolution for surfaces I and II, a fact that has been previously observed in $K$ type rough surfaces. The evolution of $\varepsilon$ on surface III is observed to be rather slower and representative of a $D$ type surface. In Fig. 4(c) the value of $\varepsilon$ calculated through Thompson's procedure furnishes 2.44 .

The values of $C_{\mathrm{f}}$ obtained through the two velocity gradient methods are shown in Fig. 5. They are also compared with a classical calculation method that makes use of von Karman's integral momentum equation.

\subsection{Temperature profile data}

The measured temperature profiles for the three different flow configurations are shown in Fig. 6. Much in the same way as with the velocity profiles, the temperature profiles are also observed to present a shift to the left when compared with data for a smooth wall. Here we just notice that, since close to the point of change in surface nature the thermal boundary layer is still in its initial state of development, a logarithmic region cannot be clearly identified in the first stations.

Fig. 6 suggests that all the procedures advanced for the evaluation of $\varepsilon$ and of $C_{\mathrm{f}}$ can be extended to the temperature profiles for the evaluation of $\varepsilon_{\mathrm{t}}$ and of $S t$.
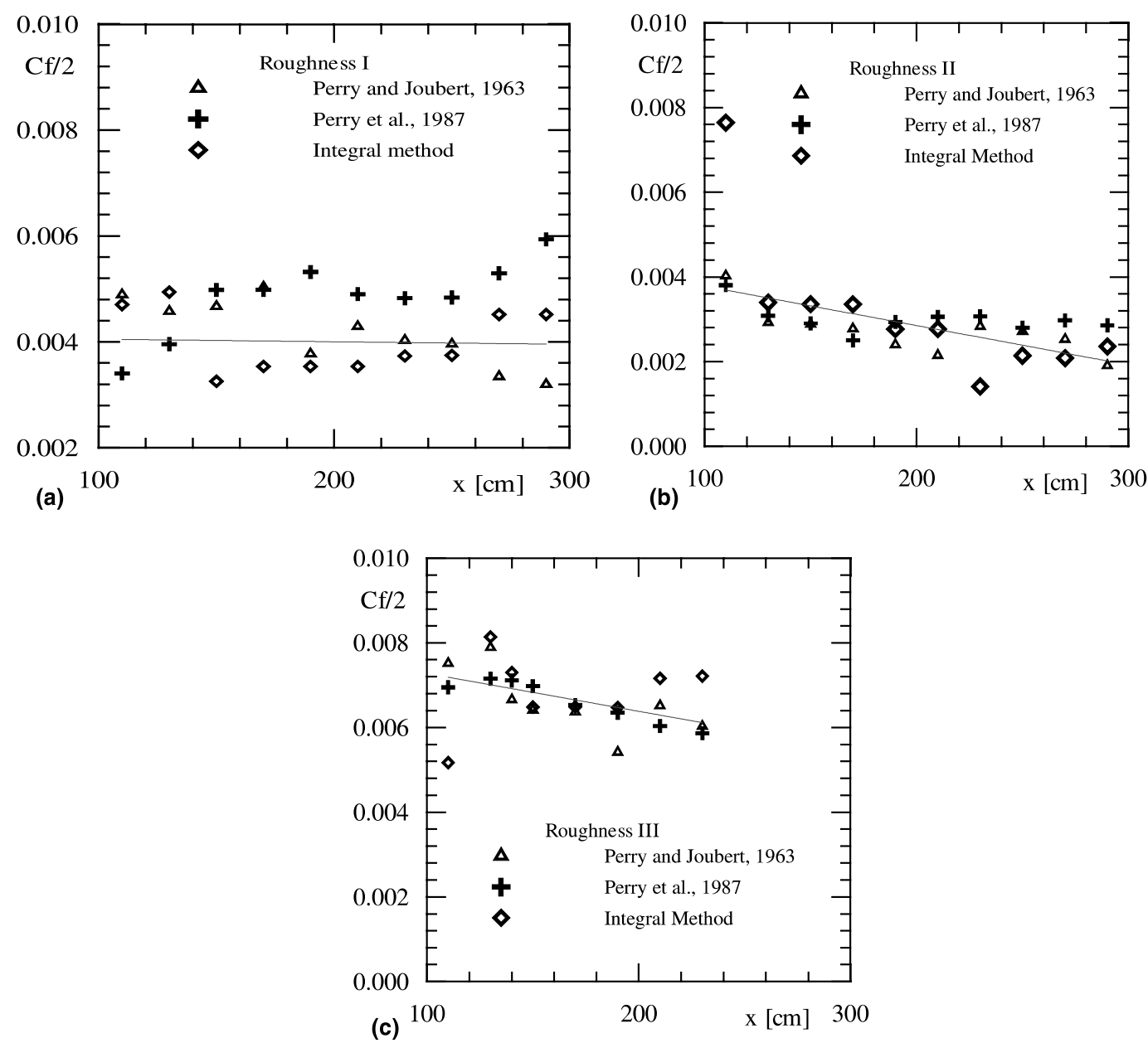

Fig. 5. Skin-friction coefficient: (a) roughness of type I; (b) roughness of type II; (c) roughness of type III. ( $\triangle$ ) [1], (+) [5]; $(\diamond)$ momentum equation. 

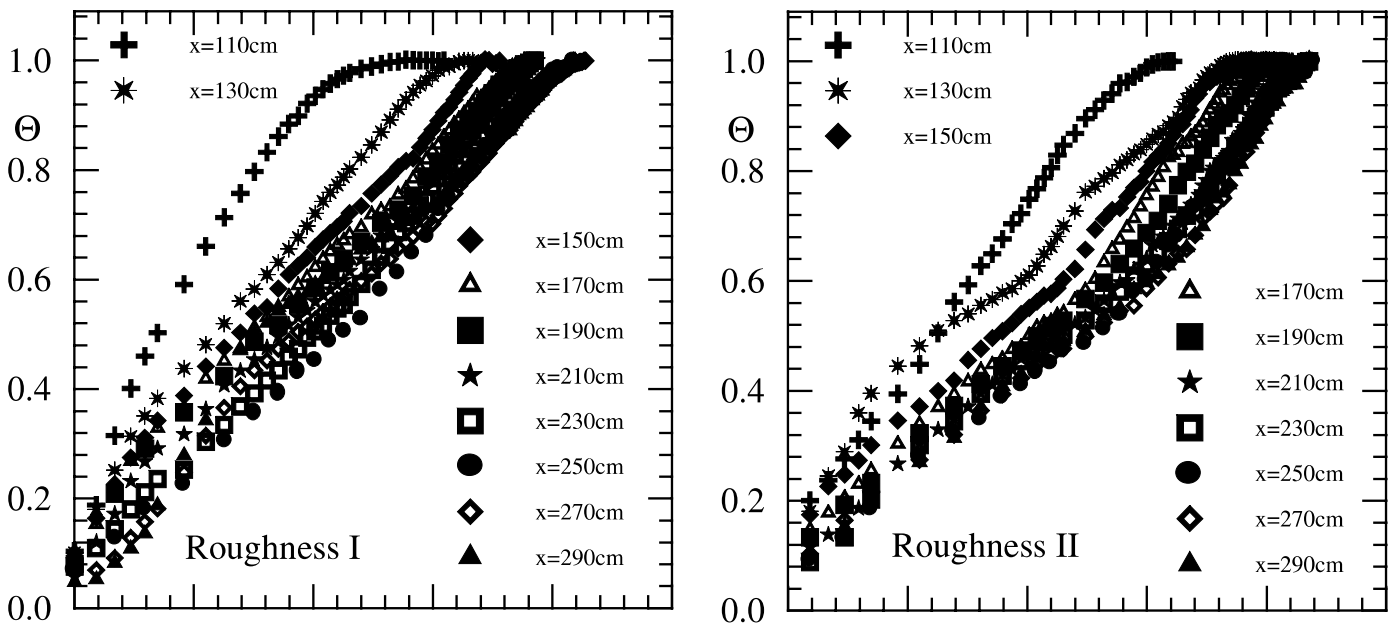

(a) 0

$2 \ln \mathrm{y}[\mathrm{mm}] 4$

(b) 0

$2 \ln \mathrm{y}[\mathrm{mm}] \quad 4$

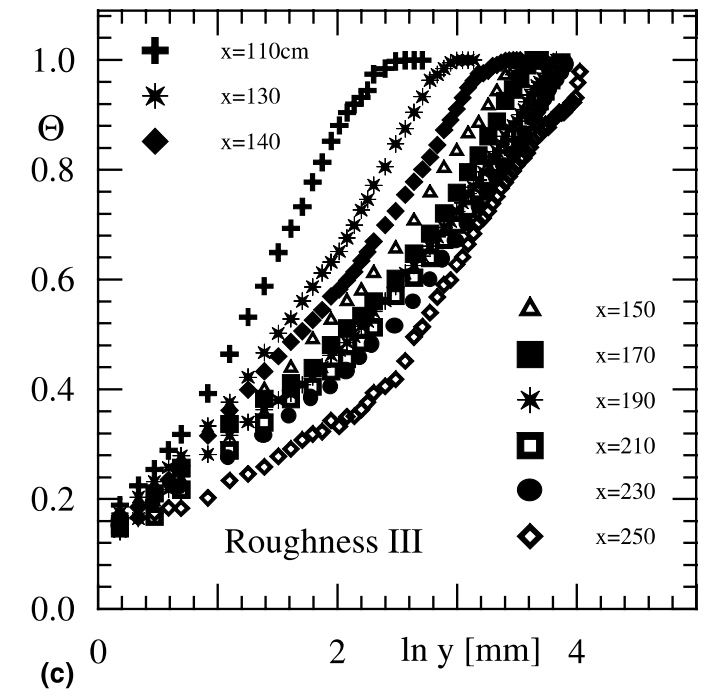

Fig. 6. Temperature profiles: (a) roughness of type I; (b) roughness of type II; (c) roughness of type III. $\Theta=\left(T_{\mathrm{w}}-t\right) /\left(T_{\mathrm{w}}-T_{\infty}\right)$.

Then, a straightforward extension of the method of Perry and Joubert [1] to the temperature profiles can be made to evaluate $\varepsilon_{t}$. The method of Perry et al. [5] could not be used here due to the low degree of development of the outer region of the temperature profile over the heated surface. Thus, a difficult characterization of the wake profile prevented us from using the cross plot method of Perry et al. [5].

Fig. 7 presents the evaluated temperature error in origin for all types of surfaces.

For surfaces of types I and III, the error in origin for the temperature profiles was systematically found to attain much higher values than the error in origin for the velocity profiles. In fact, for the total length of the heated surface considered in this work, $\varepsilon$ and $\varepsilon_{\mathrm{t}}$ approached different limiting values at the end of the test section. This is illustrated in Table 2.

For surface II, however, where relation $W / K \gg 3.0$ holds, the calculated $\varepsilon$ and $\varepsilon_{\mathrm{t}}$ are seen to approach asymptotically the same value; $\varepsilon \approx \varepsilon_{\mathrm{t}} \approx 1$.4. Moreover, the experiments show that $\varepsilon_{\mathrm{t}}$ grows at about the same rate of $\varepsilon$.

Thus, it appears that for surfaces which have a small aspect ratio, $W / K$, the error in origin for the velocity and the temperature profiles follows a different behavior with $\varepsilon_{\mathrm{t}}$ growing at a much faster rate.

Having found $\varepsilon$ and $\varepsilon_{t}$, we can now use the gradient of the log-laws to determine $S t$; this can be made through Eqs. (1)-(6). The results are shown in Fig. 8. 


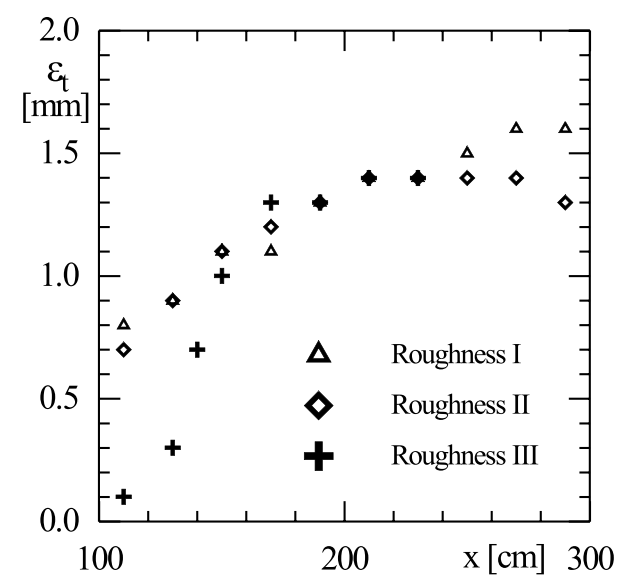

Fig. 7. Error in origin for the temperature profiles according to Perry and Joubert [1]. Roughness of types I, II and III.

Table 2

Behavior of error in origin

\begin{tabular}{lll}
\hline Type of surface & $\varepsilon(\mathrm{mm})$ & $\varepsilon_{\mathrm{t}}(\mathrm{mm})$ \\
\hline I & 1.2 & 1.5 \\
II & 1.4 & 1.4 \\
III & 0.8 & 1.2 \\
\hline
\end{tabular}

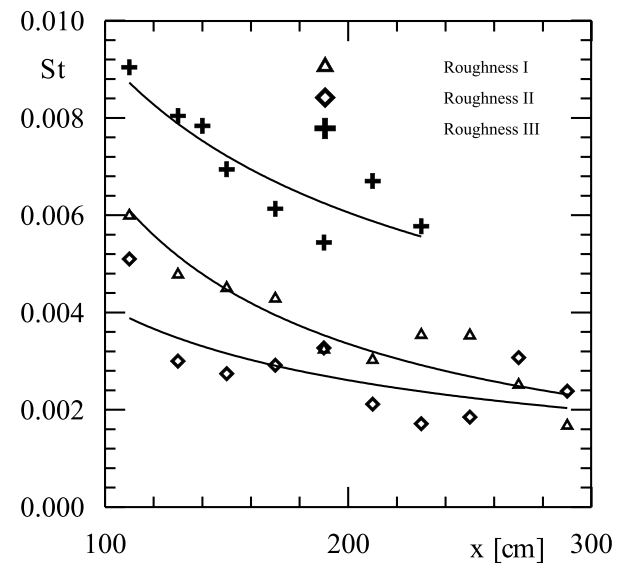

Fig. 8. Stanton number results according to Perry and Joubert [1]. Roughness of types I, II and III.

Despite the notable differences found for the evaluated values of $\varepsilon$ and $\varepsilon_{\mathrm{t}}$ for surfaces I and III, $C_{\mathrm{f}}$ and $S t$ were found to present nearly the same behavior.

\section{Final remarks}

The calculated values of $\varepsilon$ and of $\varepsilon_{t}$ were obtained through the methods of Perry and Joubert [1] and of
Perry et al. [5]. In the first method, by systematically adding an arbitrary value to the distance from the top of the roughness elements, a least square procedure was built to furnish the best discriminated straight line fit. The second method uses the universal wake profile of Coles and resorts to a cross plot of $\varepsilon$ vs $2 \Pi / x$.

In previous works, some authors (see, e.g., [19]) have expected, on asymptotic grounds, that the values of $\varepsilon$ and of $\varepsilon_{\mathrm{t}}$ would be very close. Here, we have shown that this appears to be the case for surfaces where $W / K \gg$ 3.0 , surfaces of type $K$; for surfaces of type $D$ the results differ appreciably.

Determining the error in origin has always been a difficult problem that has plagued many authors. Here we have made a comparison between $\varepsilon$ and $\varepsilon_{\mathrm{t}}$ for three different types of surfaces. Since the main objective of this work has been to assess the usefulness of Eqs. (1)(6), we have presented only mean velocity and temperature data. Measurements of turbulent quantities and a further processing of the available data will be presented elsewhere.

In completion to the work of Guimarães et al. [19], this work has shown that a working relationship between the rates of growth for the error in origin for the velocity and the temperature profiles can be established. This is a very important matter for it allows Stanton number to be evaluated directly from a proper equation which takes into account the different states of development of the velocity and the temperature boundary layers. Evidence suggests that for surfaces of type $K$ both $\varepsilon$ and $\varepsilon_{t}$ grow at the same rate. For surfaces of type $D$ this does not seem to be the case.

\section{Acknowledgements}

The present work was financially supported by the Brazilian National Research Council - CNPq - through grant no. 350183/93-7.

\section{References}

[1] A.E. Perry, P.N. Joubert, Rough-wall boundary layers in adverse pressure gradients, J. Fluid Mech. 17 (1963) 193-211.

[2] R.A. Antonia, R.E. Luxton, The response of a turbulent boundary layer to a step change in surface roughness. Part 1. Smooth to rough, J. Fluid Mech. 48 (1971) 721761.

[3] R.A. Antonia, R.E. Luxton, The response of a turbulent boundary layer to a step change in surface roughness. Part 2. Rough to smooth, J. Fluid Mech. 53 (1972) 737-757.

[4] D.H. Wood, R.A. Antonia, Measurements in a turbulent boundary layer over a d-type surface roughness, J. Appl. Mech. 53 (1975) 591-596. 
[5] A.E. Perry, K.L. Lim, S.M. Henbest, An experimental study of the turbulence structure in smooth- and roughwall boundary layers, J. Fluid Mech. 177 (1987) 437-466.

[6] J.P. Hartnett, E.R.G. Eckert, R. Birkebak, R.L. Sampson, Simplified procedures for the calculation of heat transfer to surfaces with non-uniform temperatures, WADC Technical Report, 1956, pp. 56-373.

[7] D.S. Johnson, Velocity, temperature and heat transfer measurements in a turbulent boundary layer downstream of a stepwise discontinuity in wall temperature, ASME Trans. J. Appl. Mech. 24 (1957) 2-8.

[8] D.S. Johnson, Velocity and temperature fluctuation measurements in a turbulent boundary layer downstream of a stepwise discontinuity in wall temperature, ASME Trans. J. Appl. Mech. 26 (1959) 325-336.

[9] W.C. Reynolds, W.M. Kays, S.J. Kline, Heat transfer in the turbulent incompressible boundary layer II: step wall temperature distribution, NASA Memo 12-2-58 W, 1958.

[10] W.C. Reynolds, W.M. Kays, S.J. Kline, Heat transfer in the turbulent incompressible boundary layer III: arbitrary wall temperature and heat flux, NASA Memo 12-3-58 W, 1958.

[11] D.B. Spalding, Heat Transfer to a turbulent stream from a surface with spanwise discontinuity in wall temperature, international developments in heat transfer, ASME/Inst. Mech. Engrs. Part II 439 (1961).

[12] R.A. Antonia, W.H. Danh, A. Prabhu, Response of a turbulent boundary layer to a step change in surface heat flux, J. Fluid Mech. 80 (1977) 153-177.

[13] H.W. Coleman, R.J. Moffat, W.M. Kays, Momentum and energy transport in the accelerated fully rough turbulent boundary layer, Report No. HMT-24, Stanford University, 1976.

[14] P.M. Ligrani, R.J. Moffat, W.M. Kays, The thermal and hydrodynamic behaviour of thick rough-wall turbulent boundary layers, Report No. HMT-29, Stanford University, 1979.
[15] P.M. Ligrani, R.J. Moffat, W.M. Kays, Artificially thickened turbulent boundary layers for studying heat transfer and skin-friction on rough surfaces, J. Fluids Eng. 105 (1983) 146-153.

[16] P.M. Ligrani, R.J. Moffat, Thermal boundary layers on a rough surface downstream of steps in wall temperature, Boundary Layer Meteorol. 31 (1985) 127-147.

[17] A.P. Silva Freire, M.H. Hirata, Analysis of thermal turbulent boundary layers over rough surfaces, in: Proceedings of the 3rd Brazilian Meeting on Thermal Sciences, Florianópolis, 1990, pp. 313-316.

[18] M.R. Avelino, P.P.M. Menut, A.P. Silva Freire, Characteristics of turbulent boundary layers over surfaces with abrupt variation in properties, trends in heat and mass transfer, Res. Counc. 5 (1999) 63-80.

[19] J.H.D. Guimarães, S.J.F. Santos Jr., J. Su, A.P. Silva Freire, The turbulent boundary layer subjected to a sudden change in surface roughness and temperature, in: Proceedings of the IMECE99, November 12-14, Tennessee, USA, 1999.

[20] S.J. Kline, The purpose of uncertainty analysis, J. Fluids Eng. 107 (1985) 153-160.

[21] W.L. Moore, An experimental investigation of the boundary layer development along a rough surface, Ph.D. Thesis, State University of Iowa, 1951.

[22] J. Nikuradse, Stromungsgesetze in Rauhen Rohren, V.D.I. Forshungsheft No. 361, 1933.

[23] D. Coles, The law of the wake in the turbulent boundary layer, J. Fluid Mech. 1 (1956) 191-226.

[24] R.S. Thompson, Note on the aerodynamic roughness length for complex terrain, J. Appl. Meteorol. 17 (1978) 1402-1403.

[25] P.R. Bandyopadhyay, Rough wall turbulent boundary layers in the transition regime, J. Fluid Mech. 180 (1987) 231-266. 